\title{
A Case of a Large Coronary Artery Fistula
}

\author{
Yevgeniy Latyshev, MD*
}

Jersey Shore Medical Center, USA

*Corresponding author: Yevgeniy Latyshev, MD, Jersey Shore Medical Center, 1945 NJ-33, Neptune City, NJ 07753, USA

\begin{abstract}
Coronary artery fistulas are rare anomalies found during coronary angiography and non-invasive cardiac imaging. Recent advances in understanding of the pathophysiology allow more effective surgical and percutaneous treatments. It is important to recognize and treat hemodynamically significant fistulas when appropriate before ischemia and or heart failure occurs. We present a case of coronary artery fistula that was treated conservatively after a heart team discussion.
\end{abstract}

\section{Introduction}

Coronary artery fistulas (CAF) are rare cardiac anomalies. Mostly found incidentally during cardiac catheterization. Since the increase use of computer tomography coronary angiography, prevalence of CAF increased from $0.3 \%$ up to $0.9 \%$ of congenital heart disease $[1,2]$. Here we report a case of a congenital coronary artery fistula diagnosed during evaluation of mild dyspnea.

\section{Case Presentation}

A 62-year-old male with a past medical history of hypertension, hyperlipidemia was evaluated by cardiology for mild dyspnea on exertion. Transthoracic echocardiogram showed mildly depressed left ventricular ejection fraction of $40-45 \%$, and no significant valvular abnormalities. Nuclear stress test showed mild ischemia in anteroapical segments. Subsequent cardiac catheterization showed very large normal coronary arteries with arteriovenous fistula (Video 1). Coronary computer tomography angiography (CT) showed markedly dilated $10 \mathrm{~mm}$ left main coronary artery, 8 $\mathrm{mm}$ left anterior descending artery, and a $12 \mathrm{~mm}$ left circumflex coronary arteries. Left circumflex artery was tortuous with a small fistulous track from the proximal vessel to the superior vena cava and a suspected fistula to the left ventricle. Distal branches of the circumflex along the inferior margin of the heart were also tortuous. Dilated ascending aorta at $4.5 \mathrm{~cm}$ was also recognized. After heart failure regiment was optimized, dyspnea resolved. The patient was evaluated by cardiothoracic surgery and after a heart team discussion, a decision was made to continue medical management and to closely monitor for aortic root growth. 1 year later, the patient remained asymptomatic with no further enlargement of the aortic root aneurysm.

\section{Discussion}

Coronary artery fistula was first described in 1865 by Kraus [3]. In 1963 Haller and Little described the clinical manifestation of coronary artery fistula: atria or ventricular left-to-right shunt and a large tortuous coronary artery [4]. The majority is congenital but some are acquired. Cardiac stent placement, congenital heart surgery, cardiac bypass surgery, pacemaker placement, and trauma can cause iatrogenic coronary artery fistula.

CAF can lead to various complications including myocardial ischemia, heart failure, arrhythmia, and infective endocarditis (IE). Coronary steel causes decreased perfusion distal to the coronary artery fistula. Symptoms of ischemia may occur at the time of increased oxygen demand. With left-to-right shunting, volume overload can lead to bilateral chamber dilation and pulmonary hypertension. Left-to-left shunt can cause isolated left heart volume overload and dilation, sparing pulmonary circulation. IE is another significant complication of CAF. Estimated prevalence of IE is $2-12 \%$ of reported CAF cases [5].

Invasive coronary angiography remains the gold standard for assessment of CAF. Although invasive 
coronary angiography has high spatial and temporal resolution, it is difficult to delineate the complex CAF anatomy with two dimensional images. The invasive nature of the procedure also carries risks. Computer tomography angiography offers an alternative with excellent anatomical information using multiplanar reconstruction with 3D volume-rendered imaging [6].

CAF is classified by their origin, complexity and drainage site. CAF may arise from any branch of the coronary artery system. Right coronary artery (RCA) gives rise to CAF in $60 \%$ of the cases, left anterior descending (LAD) artery in $35 \%$ of the cases, and from the right posterior descending artery (RPDA), obtuse marginal (OM) and diagonal (D) arteries in $0.5 \%$ to $1.9 \%$. Sakakibara developed an angiographic classification of the CAF. Type A CAF, proximal coronary segment dilated to the origin of the fistula, with artery distal to the fistula origin remaining normal size. Type B CAF, coronary artery dilated over the entire length of the artery $[7,8]$. The drainage site is more important and classified into two categories: coronary cameral fistulas, and coronary arteriovenous fistula. Coronary cameral fistulas drain into right and left cardiac chambers, right being more common. Coronary arteriovenous fistulas drain into any segment of pulmonary or systemic circulation. Coronary cameral fistulas dilate because of a constant blood flow from high to low pressure system [6].

Surgical or percutaneous closure of CAF is indicated for symptomatic medium to large CAF. An algorithm for fistula closure has been developed by Al-Hijji, et al. Small CAF can close spontaneously over time. Among percutaneous techniques, coils, vascular plugs and covered stents can be used to treat CAF. Transarterial approach, transvenous approach or both are feasible depending on the anatomy. Percutaneous closure of large fistulas can be complicated with stagnant flow, clot formation and propagation resulting in myocardial infarction. Surgical CAF ligation and coronary artery bypass grafting should be considered in select cases [9].

\section{Conclusion}

At times it is challenging to decide on appropriate CAF treatment especially when other cardiac abnormalities and anomalies are present. A multidisciplinary heart team discussion is necessary. Maximal medical therapy and close clinical follow up is an option before optimal timing for surgical intervention, if needed, is determined.

\section{References}

1. Yildiz A, Okcun B, Peker T, Arslan C, Olcay A, et al. (2010) Prevalence of coronary artery anomalies in 12,457 adult patients who underwent coronary angiography. Clin Cardiol 33: E60-E64.

2. Lim JJ, Jung JI, Lee BY, Lee HG (2014) Prevalence and types of coronary artery fistulas detected with coronary CT angiography. AJR Am J Roentgenol 203: W237-W243.

3. Krause W (1865) Uber den Ursprung einer accessorischen a. coronaria cordis aus der a. pulmonalis. Z Ratl Med 24: 225-227.

4. Haller JA Jr, Little JA (1963) Diagnosis and surgical correction of congenital coronary artery-coronary sinus fistula. Circulation 27: 939-942.

5. Said SA (2016) Characteristics of congenital coronary artery fistulas complicated with infective endocarditis: Analysis of 25 reported cases. Congenit Heart Dis 11: 756-765.

6. Yun G, Nam TH, Chun EJ (2018) Coronary artery fistulas: Pathophysiology, Imaging Findings, and Management. Radiographics 38: 688-703.

7. Geller CM, Dimitrova KR, Hoffman DM, Tranbaugh RF (2014) Congenital coronary artery fistulae: A rare cause of heart failure in adults. J Cardiothorac Surg 9: 87.

8. Sakakibara S, Yokoyama M, Takao A, Nogi M, Gomi H (1966) Coronary arteriovenous fistula. Nine operated cases. Am Heart J 72: 307-314.

9. Al-Hijji M, El Sabbagh A, El Hajj S, AlKhouli M, El Sabawi B, et al. (2021) Coronary artery fistulas: Indications, techniques, outcomes, and complications of transcatheter fistula closure. JACC: Cardiovascular Interventions 14: 1393-1406. 